\title{
Visual Storytelling in Advertising: A Study of Visual Storytelling as a Marketing Approach for Creating Effective Ads
}

\author{
Doaa Farouk El-Desouky* \\ Associate Professor, Faculty of Mass Communication, Advertising \& PR Department, Ahram Canadian \\ University. Egypt.
}

*Corresponding Author: Doaa Farouk El-Desouky, Associate Professor, Faculty of Mass Communication, Advertising \& PR Department, Ahram Canadian University. Egypt.

\begin{abstract}
Visual stories are a powerful and effective method of communicating ideas, wisdom, cultural knowledge and historical events. The rise of visual social media platforms has also resulted in the present economy in which the consumers welcome real-time marketing in the form of "snack able" bits of visual content. Accordingly visual storytelling has become a potent force in content marketing as brands have found it imperative to include visual content and narratives into their marketing agenda. With the rise of visual storytelling as a contemporary effective way of communicating \& marketing ideas to the public, here emerged the importance \& significance of this paper discussing visual storytelling in advertising as a marketing approach that can help creating effective \& powerful ads. The paper follows the descriptive research methods in discussing visual storytelling as a marketing approach for creating effective ads \& the demonstration of advertising samples using visual storytelling as a strategy.
\end{abstract}

Key words: Visual Storytelling, Communication, Marketing, Advertising, Media.

\section{INTRODUCTION}

Before the days of neon lights and marquee signs, business owners had to get creative when it came to advertising their establishments. In fact, wine bars in ancient Rome used to hang a bunch of vine leaves outside their door as a nod to the God of Wine, Bacchus. And when weather conditions left them with a short supply of vine leaves, barkeeps turned to bushes - inns called bush - \& bushes, still exist today.

But others didn't even have that. They had to get artistic. And with a largely illiterate population, pictorial signs were the only logical advertising choice.

Visual storytelling -- or passing on a lot of information through a relatively simple visual aid -- has been a cornerstone of marketing for thousands of years, and still what worked then works now. We see what we want and we're driven to buy it,and if anything has changed it's more of the purpose, as today's visual storytellers have a chance to lead the consumer into a whole new world of possibilities.[1] (De Meré, 2016)

\section{What IS VisUal STORYTELLING?}

By definition, visual storytelling might seem pretty straightforward - it's the art of communicating a series of messages using visual media. The concept isn't new to our cultural landscape. In fact, the phrase "A picture is worth a thousand words" was coined just after the turn of the 20th Century.[2] (Scherman, 2016)

But the concept of visual storytelling is more relevant now than ever, especially in our highly digital age in which there is a constant battle for consumers' attention.

Another definition is: "Visual storytelling involves the use of graphics, images, pictures, and videos to engage with viewers in an effort to drive emotions, engage inter-communication, and motivate an audience to action.”[3] (Shiao, 2020)

Today's visual storytelling involves the use of graphics, images, pictures and videos to engage with viewers in an effort to drive emotions and interaction. These are tactics marketers have been tuned 
into for years, but with the rapid rise in digital marketing, they're now calling upon designers to bring their messages to life. Talented designers are essential in presenting data and messaging in visual formats, which is critical to visual storytelling. Once they have all the necessary details, designers are tasked with turning information into relevant, informative, eye-catching pieces of art. Chief among these formats is video, but visual storytelling can include everything from compelling photojournalism and educational info-graphics to cutting-edge comics, GIFs and memes.

Visual storytelling isn't about pairing a piece of content with an image; it's about determining the right way to represent the information to ensure that it is compelling and relevant for the right audience. When the visual aspect is at its most powerful, the impact and performance of the content is magnified. [2](Scherman, 2016)

Visual storytelling starts with a story. A story is the telling of an event or series of events via any medium and in any way, e.g., written, verbal, with images, via TV, via radio, via social media, etc. A visual story is a story with the explicit use of visuals to convey that story.

Visuals in themselves are powerful. Images are for example much more powerful than plain text. A great story is a powerful way to communicate, and adding images to that story strengthens the message even more.

\section{THE EVOLUTION OF VISUAL STORYTELLING}

Visual storytelling has not been an overnight sensation, but instead it has been the result of a continued evolution of social media platforms, along with user and company behaviors. From the late 1990s to mid-2000s, sites such as blogging platforms, MySpace, Delicious (formerly Del.icio.us), Flickr, and Facebook created early opportunities for visual engagement. If you look closely at these platforms, you'll see that all of them were created in response to the massive flow of content on the Internet, much of which was visual.

Besides the content shared, perhaps one of the most important qualities these early social media platform leaders had was the ability to bring like-minded individuals together online. Just as today, when there are so many social media platforms to engage on, like-minded individuals always seemed to find their way into the online communities that best reflected their interests. The key then, as it is now, was to identify what brings people to a social media platform, understand what value they derive from the platform, and determine how your company could fit within those engagement best practices.

In addition to community engagement, the activity on these platforms also reinforced that users were looking for ways to easily share and organize content, imagery, visuals, and videos.[4](Walter \& Gioglio, 2014)

\section{WhY IS ViSUAL STORYTELLING So IMPORTANT?}

The telling of a story lies deep in the social behavior of human groups - ancient and modern. They dramatize social relations and problems of living, convey ideas or act out of fantasies. Thus the telling of a story requires skill. Storytelling preserved knowledge by passing it from one generation to another and this mission has continued into modern times. The early storytellers used crude images supported with gestures and vocal sounds which later evolved into language. And over the centuries as technology provided paper, printing machines \& electronic transmitters till entering the digital age, these developments directly affected the narrative arts.[5] (Eisner, 2008)

\section{Visual STORYTELling THAT MAKES THE BIGGEST IMPACT}

All forms of visual storytelling usually provide credible statistics, but it shouldn't surprise you that video is king when it comes to the most impactful format. While video may be king, it's not the only type of visual storytelling audiences want. Regardless of format, all forms of visual storytelling should adhere to a few important principles.

News Cred and Getty Images teamed up to identify what they're calling "The Four Principles of Visual Storytelling": [2](Scherman, 2016)

\subsection{Authenticity}

Immersed with so much content on a daily basis, audiences have become more sophisticated when it comes to spotting fake or sponsored content. The lesson here for designers is to remain authentic if you want to establish a personal connection with viewers. 


\subsection{Sensory}

With such a high percentage of our waking hours spent online, audiences have become somewhat desensitized to digital content. For this reason, viewers have become more and more drawn to visceral, sensory experiences. The more senses a piece of visual content is able to engage, the more memorable it becomes.

\subsection{Archetype}

Creating character-driven visual stories helps an audience establish a personal connection with the message being shared. You may be familiar with some of the most classic archetypes, such as the caregiver, the explorer, the hero and the rebel. These become powerful tools in delivering the right visuals to the audiences that will identify with them most.

\subsection{Relevancy}

A key to connecting with an audience is providing the right message at the right time through the right visual representation. Tapping into cultural events, timely occurrences and emerging trends is vital to relevant visual storytelling. [2] (Scherman, 2016)

Today, visual storytelling is no longer confined to a nostalgic review of what happened earlier in life. Visual storytelling has become a potent force in content marketing. Brands have found it imperative to include visual content and narratives into their marketing agenda since it has been shown to increase website traffic and visitor engagement. Brands effectively use visual storytelling to convey the benefits of their products or service. They have also tapped into the power of visual content as a means of converting followers into loyal patrons. When powerful visuals are created, engagement is expected to increase. Compelling and captivating visual content that is able to evoke emotions results in deeper engagement with the audience and drives prospective consumers to a decision -buying a product or service.[6] (Lile,2020)

Studies have revealed some alarming statistics about the true impact of visual content as follows: [2] (Scherman, 2016)

1. The brain processes visuals 60,000 times faster than it does plain text.

2. Content with relevant images gets 94 percent more views than content without.

3. Nearly 66 percent of updates on social media are visual content.

4. Tweets with images receive 150 percent more re-tweets.

5. Approximately 100 million internet users watch at least one online video each day.

6. 46.1 percent of consumers judge a company's credibility based on its website design.

Humans evolved over millennia to respond to visual information long before they developed the ability to read text and as Images act like shortcuts to the brain as we are visual creatures, and we are programmed to react to visuals more than to words. In the 1960s, Professor Albert Mehrabian showed that $93 \%$ of communication is in fact nonverbal. By this he meant that most of the feelings and attitudes of a message come from the facial expressions and the way the words are said, and the rest, only $7 \%$, derives from the actual words being spoken. It isn't even just the meanings of a message that are conveyed more precisely by visual information. Even issues of trust and credibility are carried by images far more so than text. [4] (Walter \&Gioglio, 2014)

In Info-graphs data visualization can transform text highlights into something compelling. Instead of trying to wrap numbers with words as numbers force readers to pause, find the digits amid the text, ponder their relationships to each other and weigh their relative values, the graphic visualization does it all at a glance.[7] (Stanton \&LaGesse, 2018)

Visual content is not only more eye-catching and memorable, but it's more engaging for its audience, which heightens its credibility, reinforces the brand and increases revenue for the company that commissioned it. This makes finding top-notch designers a high priority for companies of all kinds.

In the midst of a culture so saturated with images and videos, if you're not up-to-date on visual storytelling, you're already several steps behind competing marketeres looking to make some headway in the industry. [2] (Scherman, 2016) 


\section{The ART OF Visual Storytelling in CONTENT MARKETING}

\subsection{Telling a Story with Visual Marketing}

The human brain collects up to $80 \%$ of the sensory data received from the environment with the visual system. Appealing to the sense of vision is a powerful way to get your message across to your audience. Visuals make it easier to process difficult concepts, reduce the time it takes to learn and understand something, and it increases the amount of information that we retain. Marketers can tap into the potential of visual elements through content that is comprised of photos, videos, GIFs, and animations that attest to the value of the product or brand. [8](Olson, 2019)

Visual storytelling in the marketing context can be defined as, "A marketing strategy that leverages compelling narratives, placing the customer at the heart of the story, staged with an emotional visual media experience and effectively distributed across the buyer's journey - in order to empower customers' lives and drive business results.'[9] (Ron, 2016)

Visual storytelling helps to capture the attention of the audience and ensure that the message resonates with them. This gives the marketing company the ability to connect with the market on a deeper level and create a sense of community and loyalty with those who are drawn in by the narrative. Visuals should function like a typical story with a distinct beginning, middle, and end, accomplishing this by having a clear message and organization throughout the image.

As the brain retains 55\% more information with the addition of images instead of just words, visual mediums have the potential to make a lasting impact on the audience and convert them into loyal customers. There is a mountain of meaning that can be conveyed with very little by having a clear message marketers want to convey, a strong understanding of the audience, and purposeful execution. "Whether it is with a single photo, a video, a GIF, or animation, tell a story through visual marketing to turn viewers into customers and customers into a loyal community for the brand".[8] (Olson, 2019)

Accordingly there are some specific visual storytelling techniques in the marketing context that can help achieve those intended marketing goals: [10] (Kati, 2019)

\section{Visual StORYTELLING TECHNIQUES IN THE MARKETING CONTEXT:}

\subsection{Visual metaphors}

Visual storytelling is rarely based on words, even when you will have no other choice than to use words along with the images, they will be limited in numbers and as of consequence they should be regarded as only additions to your narrative. Different images can be used as metaphors to convey different messages instead of words such as clouds for sadness or dark times, a light bulb for a new idea, etc.

\subsection{Building Scenes by Taking Into Account Color Psychology}

Color psychology is a real thing and it helps attract the audience. It can also repulse them if colors are not being chosen right and this is what makes it especially important in the marketing process.

\subsection{Familiar Images}

Popular characters, popular faces, landscapes, products or objects, may help you capture the attention of the audience and help mentally associate those images with your brand. If you are talking to a very young audience, for instance, popular cartoon characters may be included in your stories. You can hire popular and influential faces to speak to an older audience or use products, places and items that are popular among your targeted audience as well. Giving them a sense of familiarity, each of these posts tells a story and associates in our minds with familiar instances and situations. (Figure1)
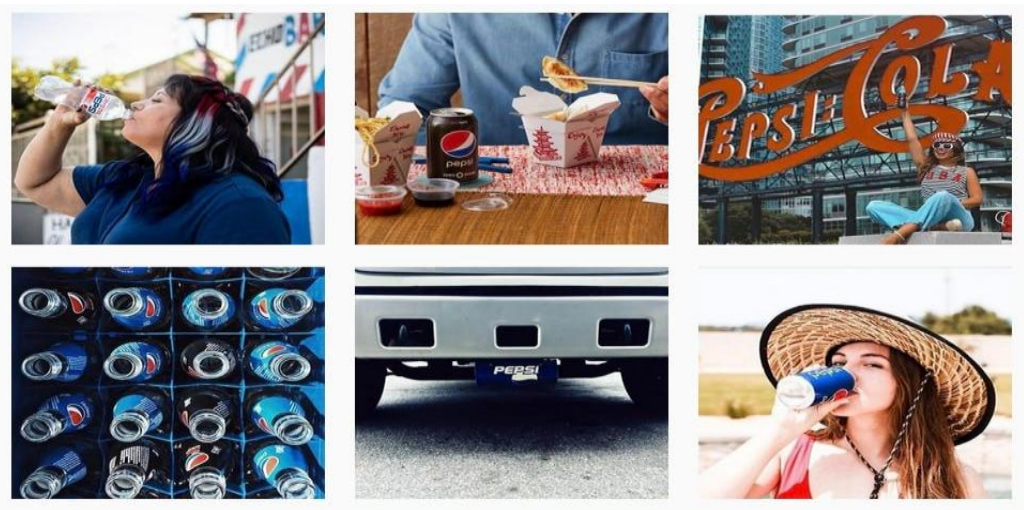

Figure1. Familiar images example from Pepsi’s Instagram profile 


\subsection{Using Light as an Advantage}

The same scene may trigger different emotions based upon the light, one of the most important elements of a visual image. While darkness and darkened landscape or environment may convey danger or mystery, people associate light and luminous spaces and objects with positive emotions. Also, the light is important outside the psychological factor, as it may outline specific items, objects or characters from your story.

For example, in a dark room, if the light falls upon a specific object, that object becomes the center of attention, the main focus of the viewer. There are a lot of properties the quality of an image depends upon. You need to take care of the perspective, the rule of thirds and at the same time the framing. They are all tied to light and lightning. (Figure 2)

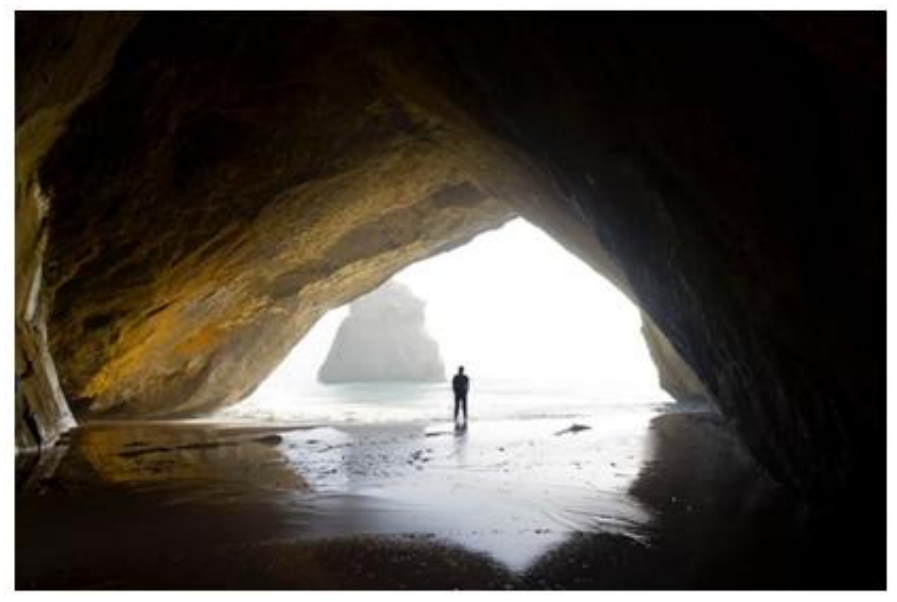

Figure2. Using light as an advantage in visual storytelling

\subsection{Telling the Whole Story}

Every story should have three main parts: beginning, middle, and end. The last one is essential as it gives closure to it and convinces the audience to act upon what you have asked from them, whether it is a follow-up click, a product check, a newsletter subscription or even a buy.[10] (Kati, 2019)

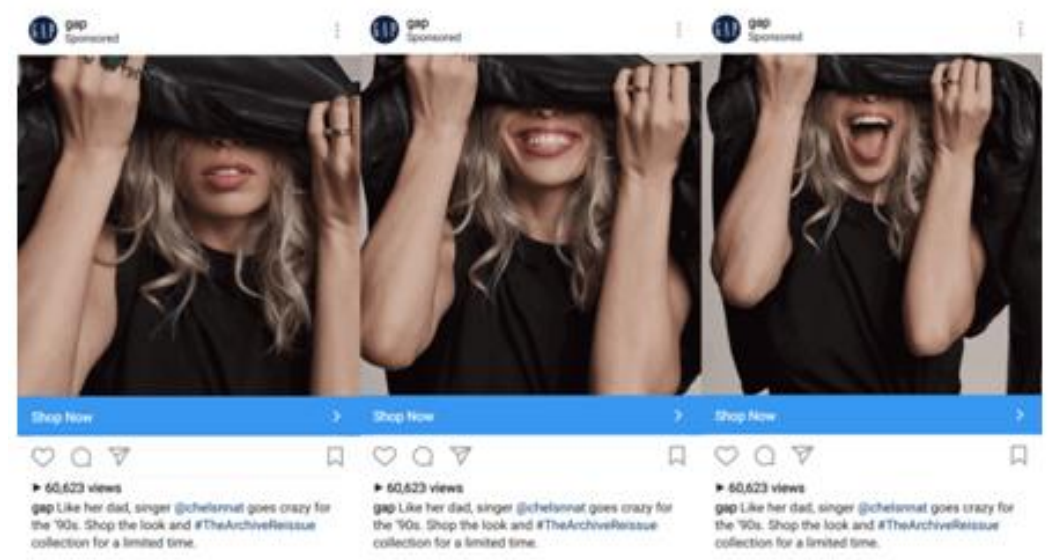

Figure3. A moving photo-shoot by Gap

Social networks like Facebook have a clever ad slideshow tool that can really make ad campaigns stand out. That's exactly what Gap did in the example above (Figure 3). The brand took three still images from a video they created and pieced together a moving photo shoot. The story here is simple and to the point, yet creative. [11] (Marsh, 2018)

Another example comes from foodsided.com, and more especially, from an article that talks about the most popular types of pies that the Americans cook and eat for Thanksgiving. (Figure 4)

The visual chart featured in the article tells a visual story and stands by itself as a great and valuable visual content item. A chart like this can be shared on all social media platforms and used as a visual marketing asset. [10](Kati, 2019) 


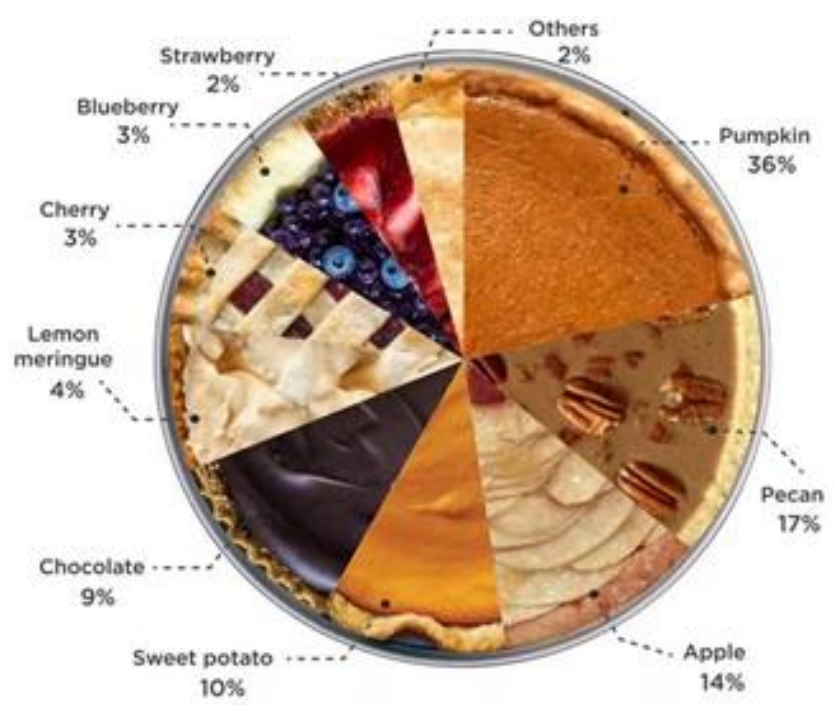

Figure4. A visual chart by foodsided.com

\section{VISUAL STORYTELLING IN ADVERTISING}

Creating images that make the viewer think and feel a certain way is big business to advertising as getting the right visuals is at the very heart of business branding. In 2001, Pepsi budgeted over $\$ 1$ billion on its image, and Coca-Cola budgeted $\$ 1.4$ billion for its image in the same year.

Communication is a two-way process, and marketers have to be as sure of their audience as they are of the images they are crafting to reach them. The right graphics can persuade, relate, and influence decisions on an emotional and subconscious level.

Images are powerful tools, as we are becoming an ever-more visual culture. Empathizing in the intended way to symbols and images is reliant on a shared cultural identity-that is, on an understanding of who your audience is. Different cultures, and even different demographics, will process symbols differently. Whereas images such as star-spangled flags and eagles can appeal to an American sense of patriotism, the same effect may be achieved by a maple leaf, a lion, or a kangaroo in other countries. And what appears strong and decisive to one audience could seem aggressive to another. Creating the right image to convey a message relies on understanding whom you are communicating with and how they will react to it.

This data is precisely why visual storytelling allows companies a strong opportunity to take these existing behaviors and cultivate content focused on harnessing consumer engagement versus broadcasting messages. Visuals draw immediate attention to a post about a topic and can generate shares, traffic, lead generation, thought leadership, and expertise. Visual storytelling can also help companies achieve earned media at scale. [4] (Walter \&Gioglio, 2014)

\section{EXAMPLES OF THE USE OF VISUAL STORYTELLING IN ADVERTISING}

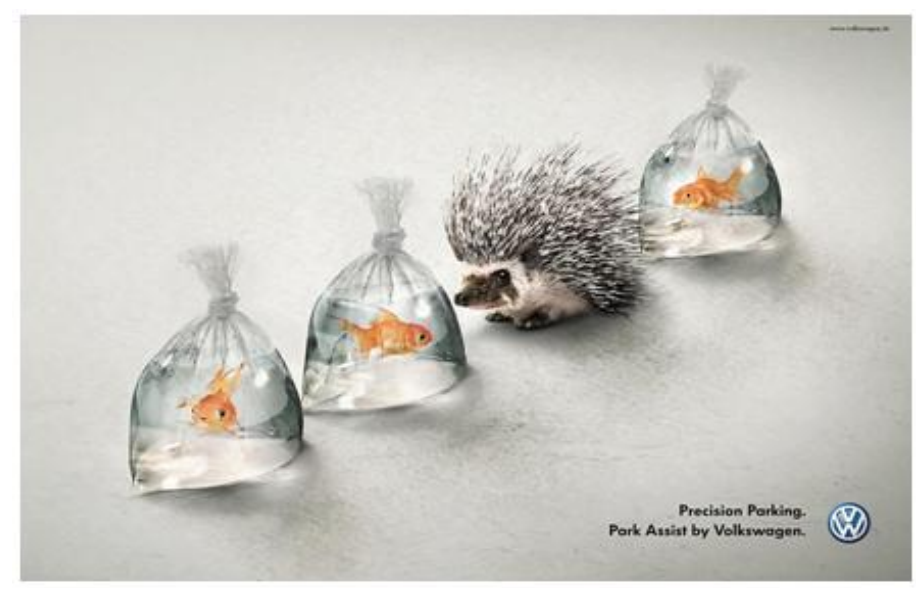

Figure5. An ad by Volkswagen 
In this ad by Volkswagen audiences can relate to the situation of parallel parking and understand the high stakes of the porcupine not hitting the goldfish bags as we know that goldfish cannot live outside of water and porcupines are pointy. (Figure 5)

This visual display grabs the attention of the audience and the absurdity of the situation appeals to our sense of humor. Volkswagen conveys the value of their Park Assist feature through portraying the story using imagery the audience understands in a humorous way that both grabs the attention of viewers and is memorable. As simple as it is, it manages to tell the whole story and convince the viewer that he has made a good choice or that he is about to make such a choice if they choose to become customers. [8] (Olson, 2019)

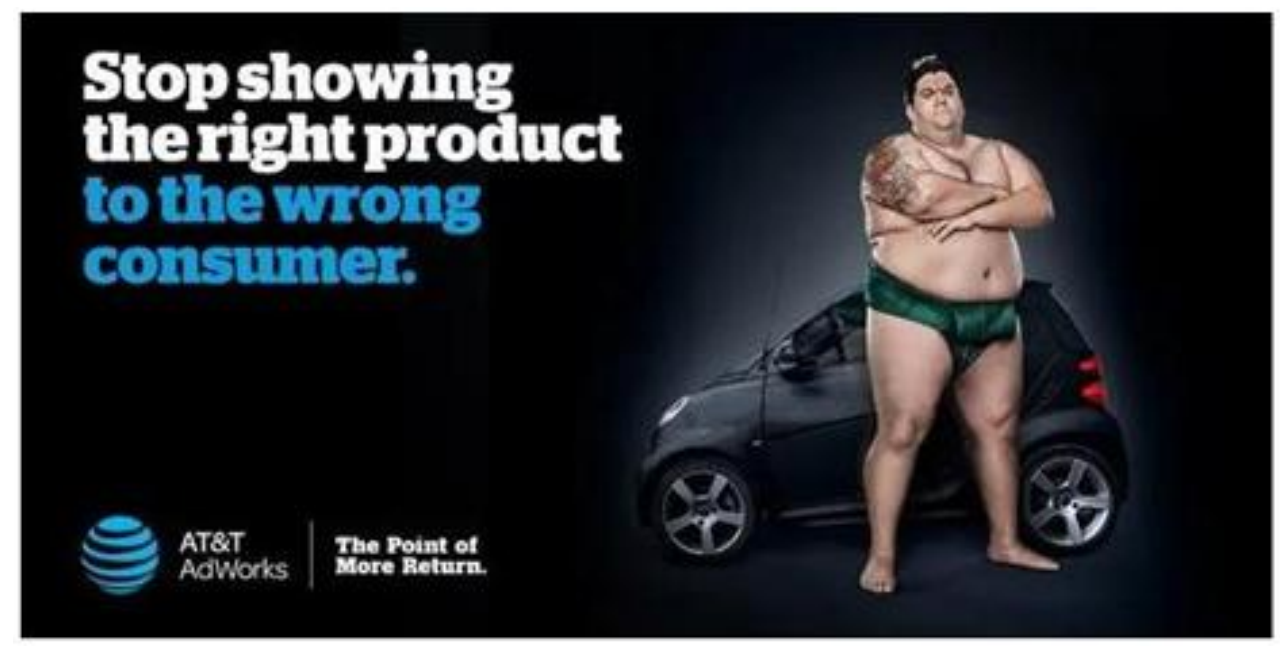

Figure6. An ad by AT\&T Ad Works

It's easy to create display ads that have an image plus supporting text. But most brands are already doing that. Share a funny story with your visual instead and you'll capture attention from anyone browsing between your ad and your competitor's.

The visual of the Sumo standing next to a tiny car tells a funny story (Figure 6) -- that it's a waste to show the "right product to the wrong consumer".[12] (Ciccarelli, 2020)

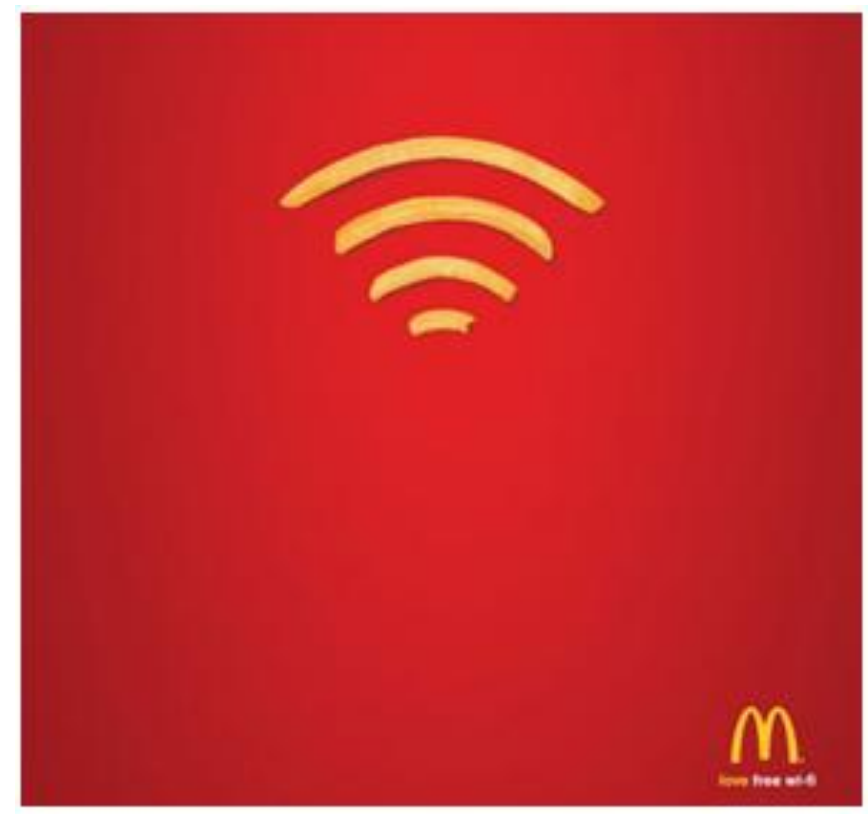

Figure7. Free Wi-Fi ad by McDonalds

This advertisement for McDonalds in (Figure 7) is another example of using storytelling in advertising where fries are used in the ad on a plain red background (one of the brand's official colors) to form the Wi-Fi signal, advertising free Wi-Fi service in McDonalds restaurants. [13] (Bryson, 2012) 


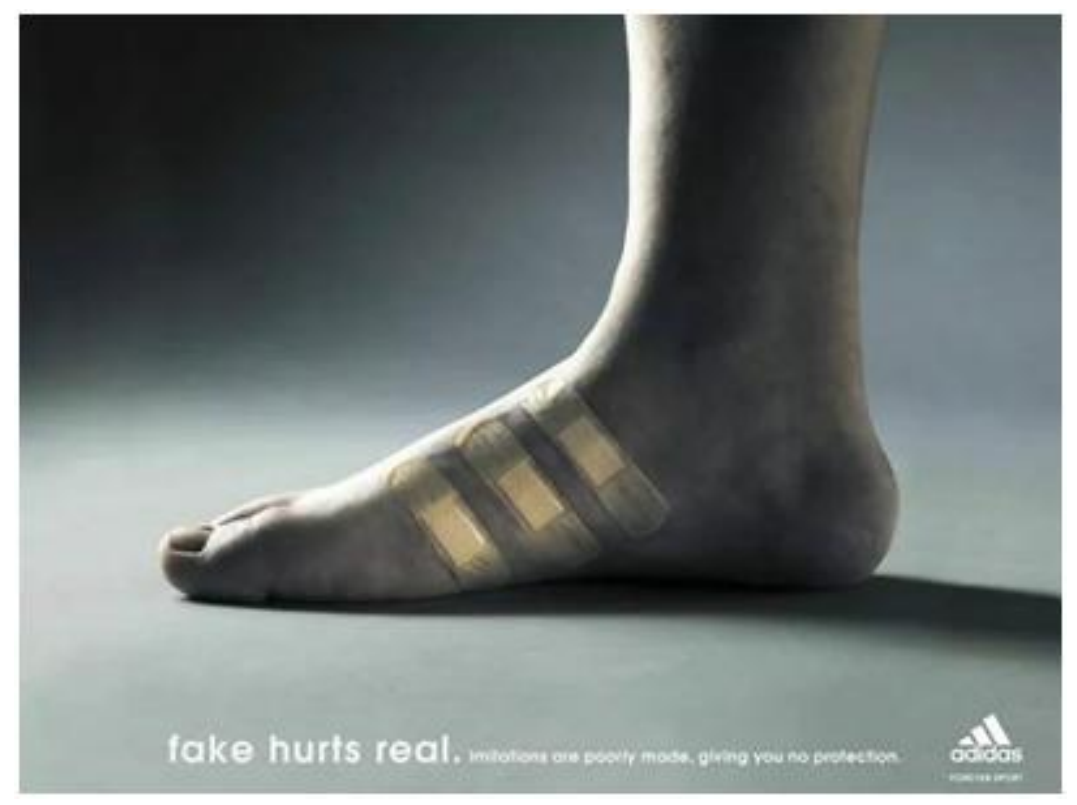

Figure8. "Fake Hurts Real” ad by Adidas

Another example is the Adidas advertisement "Fake Hurts Real" which was used by Adidas as a warning from using fake products. (Figure 8)

This smart ad by Adidas shows how imitations are harmful for both the company and the buyers. The ad implies that this person got injured feet from wearing shoes that were not original Adidas brand. This ad creates a visual story of that person especially with the use of the bandages that tells the whole story, plus giving an indication of the Adidas logo which is covering the foot in a message that Adidas will be the cure. (pinterest.com, 2019)

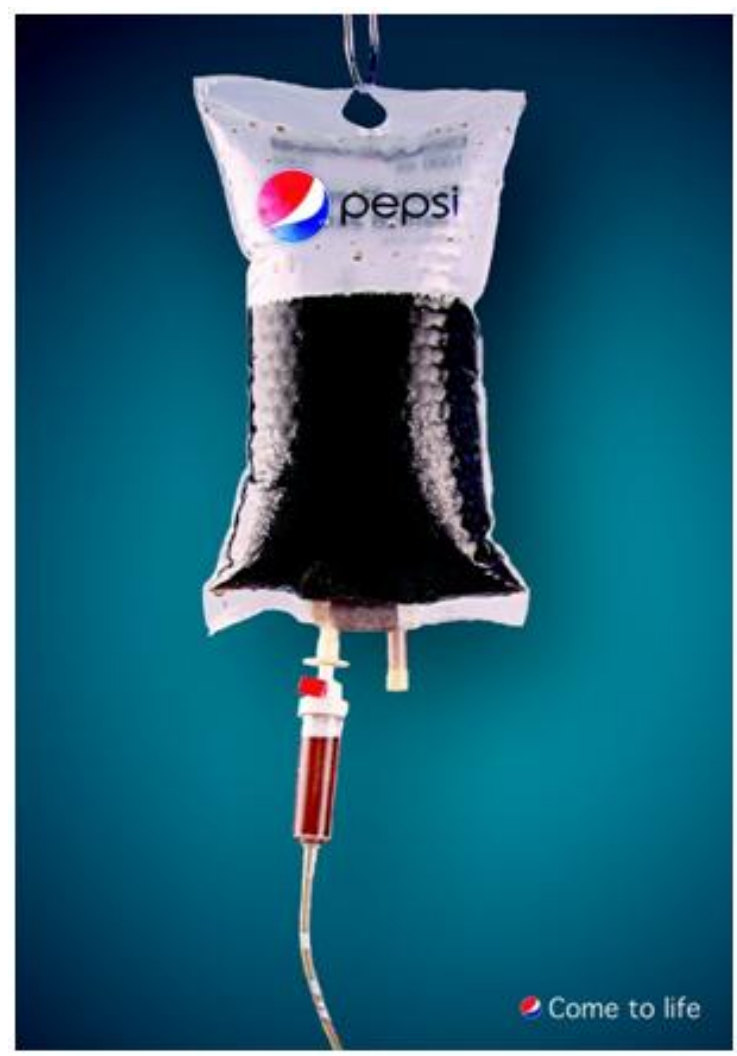

Figure9. "Come to life" ad by Pepsi

One of Pepsi sample creative adsvisualizes its product as a blood supply (Figure 9). The ad catches the audience attention \& evokes emotional connection with people presenting Pepsi drink as a source of life, using a simple caption "Come to life". (aterient.com, 2010) 


\section{How to Use Visual Storytelling EfFECTIVELY IN ADVERTISING}

\subsection{Identify The Story}

The first step to any sort of storytelling marketing is to figure out the story to convey. The messaging needs to be solidified first to create a clear purpose for the marketing content created. Make sure that the story is relevant to the audience and customer-centric so they will be able to connect to it. It is essential to show how the brand will help them become better off. To strengthen this brand story, think about what motivates the audience and relate that to what is at the core of the brand.

\subsection{Understand the Audience}

To tell this story with only an image or short progression of images, the past experiences of the audience become vital to setting up the narrative. They should be able to understand the situation and setting that you are trying to convey with ease as they have experienced those same conditions themselves or have been exposed to them before through friends, family, or other media sources. Without a large amount of words to explain what is going on, visual archetypes can be a powerful tool.

\subsection{Utilize Imagery that People will Understand its Meaning in a Memorable Way}

When it comes to storytelling in advertising, there are two big things we want to focus on: emotional impact and reliability. In other words, when you're telling your brand's story, you need to tell it in a way that will connect you with your target audience. The more people connect with a story, the more they remember it, so creating connections with your stories will help create lasting bonds and loyalty between your customers and your business. [14] (Gotter, 2017)

Take advantage of characters, objects, or places that have outside connotations to express more meaning using less. [8] (Olson, 2019)

\subsection{Choose the Suitable Platform with the Type of Visual Content}

When using it right, storytelling can strengthen every campaign and social media effort. This includes videos, which are perhaps the most effective type of media for storytelling. Videos can easily be embedded and shared across any site or platform of a business. Other types of media are also excellent platforms for visual storytelling, including blog posts, press releases, and even short social media posts. These social media posts can run as ads, building valuable brand awareness in an authentic way. [14] (Gotter, 2017)

\section{CONCLUSION}

Visual storytelling is a very powerful way to communicate. That's why every professional and organization that has to communicate, needs to use visual storytelling if they want to communicate successfully. Visual stories are a powerful and effective method of communication. Storiesparticularly specific, highly detailed stories - are the best way to create emotional connections and long-lasting bonds with target audience. They're memorable, and they're effective. Whether it's telling the brand's story through a video or a poster or a quick social media post, storytelling can all make a difference in helping build the business. And visual storytelling in advertising is a powerful approach and as long as the approach is chosen wisely, it is more likely to be successful. Visual storytelling achieves a lot; more commitment, emotions, better information flow, and more attention and in the end more customers. Building an interactive community without narrative images will be very difficult today because people love stories and they love pictures. Advertisers, in order to be able to assert successfully with their companies today, they should master the language of visual storytelling. Storytelling is itself a very effective instrument - but telling stories visually through pictures, that's the fine art of marketing.

\section{REFERENCES}

[1] De Meré, N.E. The Power of Visual Storytelling: 15 Stunning Examples to Inspire You"[Internet]. 2017 July 28. Available from: https://blog.hubspot.com.

[2] Scherman, J. What is Visual Storytelling? The Growing Trend in Multimedia Design.[Internet].2016May 30. Available from: http:/www.rasmussen.edu.

[3] Shiao, D. 3 Stunning examples show visual storytelling at its best. [Internet]. 2020 March25. Available from: http://www.contentmarketinginstitute.com. 
[4] Walter E., Gioglio J. The Power of Visual storytelling, How to use visuals, videos \& social media to market your brand), McGraw Hill Education, USA, 2014, p.9, 10, 16,17,18,21.

[5] Eisner W. Graphic Storytelling and Visual Narrative, Will Eisner Instructional Books, Will Eisner Studios, Inc., USA, 2008, p.7, 8.

[6] Lile, S. 10 Visual storytelling rules every digital marketer needs to know, [Internet]2017. Available from: http:/www.visme.com.

[7] Stanton L., LaGesseD., Superpowers of Visual Storytelling, LaVidaCo Communications, 2018, p.2, 3.

[8] Olson S. Telling a story with visual marketing. [Internet] 2019 March 7. Available from: http://www. Magicflightstudios.com.

[9] RonS. What is visual storytelling? And why marketers should care. [Internet] 2016 September 8.Available from: https://www.visualstorytell.com.

[10] Kati, R. The Art of Visual Storytelling in Content Marketing, [Internet]2019May 23. Available from: https://robertkatai.com.

[11] Marsh, Rowland. Visual storytelling for paid social. [Internet]2018 May 6.Available from: https: //www.enchantagency.com,

[12] CiccarelliD. What Makes Visual Storytelling Marketing So Effective?[Internet]2020, Available from: https://sumo.com,.

[13] Bryson, E. McDonalds Wi-Fi Ad.[Internet]2012October 7. Available from: https://elizabethbryson. wordpress.com.

[14] Gotter, A. Storytelling: The Key to Effective Advertising. [Internet]2017 August 22.Available from: https://www.disruptiveadvertising.com.

\section{AUTHORS’ BIOGRAPHY}

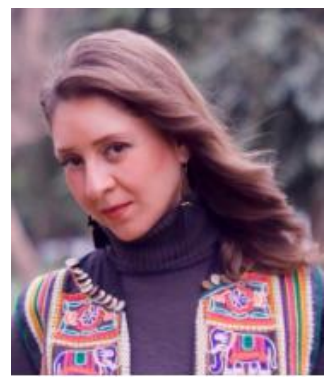

Dr. Doaa Eldesouky, Associate Professor of Advertising and a Visual Artist. Graduated from the Faculty of Applied Arts - Helwan University, Egypt with honors \& was appointed as a lecturer in the Advertising department. Had her Ph.D degree in advertising in 2006. Has several art writings \& scientific articles in the field of advertising \& visual arts. Participating in the art movement since graduation till now in the fields of drawing, painting $\&$ graphics and has a number of solo \& group - local \& international - exhibitions. Founder of AWAN Contemporary Art Space in Cairo, Egypt.

Citation: Doaa Farouk El-Desouky. "Visual Storytelling in Advertising: A Study of Visual Storytelling as a Marketing Approach for Creating Effective Ads" International Journal of Humanities Social Sciences and Education (IJHSSE), vol 7, no. 10, 2020, pp. 118-127. doi: https://doi.org/10.20431/2349-0381.0710015.

Copyright: (C) 2020 Authors. This is an open-access article distributed under the terms of the Creative Commons Attribution License, which permits unrestricted use, distribution, and reproduction in any medium, provided the original author and source are credited. 